\title{
Characteristics and retention of emergency department patients who left without being seen (LWBS)
}

\author{
Nathan Roby ${ }^{1} \cdot$ Hayden Smith ${ }^{2,4} \cdot$ Jonathan Hurdelbrink ${ }^{2,3,4}\left(\mathbb{0}\right.$. Steven Craig $^{2,4} \cdot$ Clint Hawthorne $^{2}$. \\ Samuel DuMontier ${ }^{2,4} \cdot$ Nicholas Kluesner $^{2}$
}

Received: 22 February 2021 / Accepted: 24 May 2021 / Published online: 13 June 2021

(c) Società Italiana di Medicina Interna (SIMI) 2021

\begin{abstract}
A retrospective observational study was conducted for patients 18 years or older presenting to a Midwestern emergency department (ED) in the United States during February 2019-January 2020 to characterize associated subsequent care utilization in patients who left the ED without being seen. Patients were classified as left without being seen (LWBS) based on documented ED disposition. The healthcare system's records were reviewed for any associated utilizations within 3 weeks following the initial ED encounter. During the study period, 45,456 patients presented to the ED, with 2269 (5.0\%) classified as LWBS. The median documented time until patients left the ED was $112 \mathrm{~min}$. Of these patients, $1257(55.4 \%)$ had a subsequent encounter within the health system within 3 weeks and $920(73.2 \%)$ of these visits were determined to be related to the LWBS chief complaint. These visits included $67.5 \%$ of patients returning to ED or hospital, $27.5 \%$ to primary care or an urgent care clinic, and 5.0\% to a specialty or other provider appointment. Of patients returning to ED, 78.1\% did so within $72 \mathrm{~h}$. Patients without a subsequent health system associated encounter tended to be younger, female, non-White, and present with possible lower-acuity chief complaints. At least one-half of LWBS patients sought care related to the concerns by a health system provider within 3 weeks of the initial encounter within the same system. The high prevalence of ED returns within a narrow turnaround window highlights a missed opportunity to provide services to these patients during their initial encounter.
\end{abstract}

Keywords Hospital emergency services $\cdot$ Left without being seen $\cdot$ Patient retention

\section{Introduction}

\section{Background}

The Emergency Medical Treatment and Labor Act (EMTALA) was introduced in the United States in 1986 and states that patients are entitled to a medical screening/ examination by a qualified provider when presenting to an

Nicholas Kluesner

nicholas.kluesner@unitypoint.org

1 Carver College of Medicine, University of Iowa, Iowa City, IA, USA

2 Emergency Medicine, UnityPoint Health-Des Moines, Des Moines, IA, USA

3 College of Pharmacy and Health Sciences, Drake University, Des Moines, IA, USA

4 Medical Education, UnityPoint Health-Des Moines, Des Moines, IA, USA emergency department (ED). This examination includes diagnosis, stabilization, and treatment of any emergent medical concerns and precludes referral to an urgent care or primary care office before ED evaluation [1]. Given the wide range of concerns that patients may present with, personnel working in the ED attempt to identify and triage patients based on their acuity. Unfortunately, when coupled with high patient volumes and/or acuity, this process can create substantial wait times for many patients-especially those with lower acuity of illness $[2,3]$.

While waiting for care in the ED, patients may opt to leave before being evaluated by a qualified provider. These patients are commonly referred to as 'left without being seen' (LWBS). In the literature, Johnson et al. interviewed 72 LWBS patients and found $77 \%$ of these patients left due to wait time, $12 \%$ due to concern self-resolving, and $11 \%$ for reasons such as staff demeanor, boredom, or other [4]. While this research highlights some of the reasons a patient may choose to leave the ED, the impact of LWBS 
rates on subsequent healthcare utilizations and patient retention has not been well studied.

\section{Importance}

The rate of patients who LWBS from an ED is a metric that represents the accessibility of emergency care $[5,6]$. Given the wide range of reasons why patients may leave without being seen, it is unclear if leaving necessarily impacts the patient's health or their subsequent access of medical care in a particular healthcare institution. For example, if patients presenting to an ED choose to leave without being seen, they may seek care elsewhere within the network or potentially go without care. A particularly problematic scenario is the patient who leaves without being seen with an undiagnosed and untreated emergent medical condition may experience adverse health outcomes due to the delay [7, 8]. However, if patients present to an ED with a lower-acuity complaint and leave to seek care at another facility or because their concern has self-resolved, their leaving should have no direct impact on their health outcomes. Similarly, patients who present with lower-acuity complaints may come to the ED if it is the only available option given the day/time but would otherwise have sought treatment from a primary care provider or in an urgent care setting. For patients in these scenarios, they may be less likely to seek subsequent care as it simply may not be necessary. Because of this, a greater understanding of LWBS patients would be helpful in determining how common these various scenarios are for patients and for institutions which track LWBS due to their concern for accessibility-to-emergency care and the financial implications of patients' subsequent patronage.

To this last point, LWBS patients in the United Stateswhich emphasizes a private institution-based business-model for healthcare delivery-also represent a unique population of patients who may seek care at an alternative healthcare institution in a competitive healthcare marketplace; because of this, it is certainly possible for LWBS patients to directly impact the financial situation for EDs and health systems. For these reasons, a greater understanding of the characteristics of LWBS patients, the frequency of their retention within the same organization, and the care they subsequently receive is beneficial to EDs, especially EDs in the United States.

\section{Goal of the study}

To characterize associated subsequent care utilization within a healthcare system for patients who left the ED without being seen.

\section{Methods}

\section{Study design and setting}

A retrospective observational study was conducted involving the review of electronic medical record (EMR) data for patients 18 years or older presenting to a Midwestern ED in the United States between February 2019 and January 2020. The facility is a level I trauma center in an urban community-setting, consisting of 41 acute ED beds and an annual adult ED volume of 45,000 visits. The ED is part of a multi-state health system, with all facilities using EPIC (Epic Systems Corporation, Verona, WI) as the centralized EMR. The local community also has five other public EDs, three of which are not in the health system and had inaccessible EMRs.

\section{Study protocol}

Charts were reviewed for patients with an ED disposition of LWBS and reviewed for any ED, hospital, or clinic visits within the same health system during the 3 weeks following the initial ED encounter. The health system includes a network of clinics and two additional smaller EDs in the immediate area; while patient records include visits to these other facilities, subsequent visits to other non-health system local clinics/EDs could not be captured. The first documented subsequent visit in the health system within the 3-week period was recorded and reviewed by study personnel to determine whether the care was associated with the reported chief complaint at the initial ED LWBS encounter. These visits were then categorized into three groups: returns to the ED; family medicine/urgent care visits; or specialty/other provider appointments. Also documented was whether the patient was admitted to one of the three local network hospitals within the reviewed 3 -week period, as well as if those with a subsequent ED visit did so within $72 \mathrm{~h}$.

Recorded patient data included demographic information such as age, gender, and race, as well as individual wait times and the presenting ED chief complaint. Chief complaints were originally recorded as a free text field and were reviewed and collapsed into nine categories: abdominal pain/gastrointestinal (GI); chest pain/dyspnea; gynecological/genitourinary (Gyn/GU); HEENT (head, eyes, ears, nose, and throat); immune response/infection; injury/musculoskeletal pain; neurologic/headache; psychiatric; and other. Categories were established by the research team and were comparable to those used in prior research $[10,11]$. When a nursing triage assessment was conducted, emergency severity index (ESI) scores (i.e., 
1-5; lower scores representing greater severity) and pain scores (i.e., 10 point analog scale, higher scores representing greater pain) were collected.

\section{Outcome}

Primary outcome was an associated local healthcare system visit within 3 weeks of the LWBS encounter.

\section{Data analysis}

Patient and encounter data are presented using descriptive statistics, with continuous variables presented as measures of central tendency and dispersion, while categorical data are presented as counts with percentages. Comparisons between LWBS patients with and without an associated encounter were examined using generalized linear regression modeling with a binomial distribution and logit link. Models controlled for all other reviewed covariates. Estimates are reported as adjusted odds ratios (aOR) with $99 \%$ confidence intervals (CI). Lastly, an exploratory analysis was conducted to examine the possible relationship between daily numbers of LWBS patients versus ED daily census and counts of daily ED inpatient boarding using multiple linear regression. Visualizations were generated via individual heatmaps of LWBS counts, ED census, and ED inpatient boarding by day of the week versus week of the year. This study received Institutional Review Board approval.

\section{Results}

There were 45,456 ED encounters at the study hospital during the 1-year reviewed period, with 2269 (5.0\%) of these patients having an ED disposition of LWBS. Descriptive information for these patients is provided in Table 1. Of note, 302 (13.3\%) of these LWBS patients left the ED prior to receiving an initial nurse triage assessment and did not have ESI scores, pain scores and vital sign data. Such patients registered with clerical staff at the ED entrance, but due to multiple simultaneous ED check-ins had to wait to see the triage nurse and left prior to this initial clinical assessment being recorded. These likely represent the busiest times in the ED, as the standard practice is to take the checked-in patient immediately to the triage room for nursing assessment. The LWBS encounters included 2098 unique patients, with 112 having 2 encounters and 26 having more than 2 encounters during the sample period.

Of patients seen in the ED, median wait time was 9 min [interquartile range (IQR): 0, 50]. In patients who left the ED without being seen, the median documented time until the patient left was 112 min (IQR: 58, 177). Compared to patients who did not leave the ED, LWBS patients were more likely to be younger, female, Black, and arrive at the ED on weekdays between 8:00 AM and 5:00 PM; however, Hispanic patients were less likely to leave without being seen. While undocumented/unknown race was also significant, this could be due to LWBS patients leaving before all demographic information could be collected. Finally, the chief complaint category of abdominal/GI pain had the greatest association with LWBS status compared to the other categories.

\section{Main results}

Of the 2269 LWBS patients, 1257 (55.4\%) were found to have a subsequent visit within the same health system during the next 3 weeks, whereas 1012 (44.6\%) did not; among those individuals with subsequent visits, $920(73.2 \%)$ were identified as having the subsequent visit associated with their initial LWBS encounter based on comparable chief complaints (Table 2). When examining these first subsequent visits in greater detail, $67.5 \%$ of patients returned to the $\mathrm{ED}$, $27.5 \%$ visited a family medicine or urgent care clinic, and $5.0 \%$ went to a specialty or other provider appointment in the health system. Furthermore, $52.7 \%$ of the 920 patients were considered to have returned to the ED within $72 \mathrm{~h}$ with a similar chief complaint, while $13.9 \%$ were admitted to the hospital for an associated concern at some point during the subsequent 3 weeks.

These 920 patients with an associated visit in the health system during the subsequent 3 weeks were compared with the 1349 LWBS patients who did not have a documented associated visit (Table 3). Older LWBS patients were more likely to have an associated subsequent visit (aOR: 1.08; 99\% CI 1.01, 1.15), while patients who were Black (aOR: 0.68 ; $99 \%$ CI $0.50,0.93$ ), came to the ED during the weekend (aOR: $0.63 ; 99 \%$ CI $0.45,0.89$ ) or presented with a chief complaint of HEENT (aOR: 0.41; 99\% CI 0.19, 0.87) were less likely to have an associated visit during the following 3 weeks.

Among all LWBS patients, 485 (21.4\%) had an associated return visit to the ED within $72 \mathrm{~h}$ of the initial ED encounter (Table 4). When comparing these patients with all other LWBS patients, differences were comparable to those regarding associated subsequent visits. Specifically, patients who were relatively older (aOR: 1.09; 95\% CI 1.01, 1.18) were more likely to return to the ED for an associated concern within $72 \mathrm{~h}$; however, patients who initially came to the ED over the weekend (aOR: $0.64 ; 95 \%$ CI $0.41,0.97$ ) and initially presented with a chief complaint of HEENT (aOR: $0.30 ; 95 \%$ CI $0.10,0.86$ ) were less likely to have an associated return to the ED.

In total, 128 LWBS patients were admitted into a hospital within the same health system during the 3 weeks following the initial ED encounter. The median length of stay for these 
Table 1 Characteristics of emergency department (ED) encounters stratified by left without being seen (LWBS) status, $n=45,456$

\begin{tabular}{|c|c|c|c|}
\hline Characteristics & $\begin{array}{l}\text { LWBS } \\
n=2269(5 \%)\end{array}$ & $\begin{array}{l}\text { Seen in ED } \\
n=43,187(95 \%)\end{array}$ & $\begin{array}{l}\text { Adjusted odds ratio } \\
(99 \% \mathrm{CI})\end{array}$ \\
\hline Mean age (years) & 41 (SD: 18) & 51 (SD: 21) & $0.78(0.75,0.80)^{\mathrm{c}}$ \\
\hline Male & $832(36.7 \%)$ & $19,043(44.1 \%)$ & $0.84(0.75,0.95)^{\mathrm{c}}$ \\
\hline White (race) & $1560(68.8 \%)$ & $34,045(78.8 \%)$ & 1.00 (referent) \\
\hline Asian (race) & $35(1.5 \%)$ & $735(1.7 \%)$ & $0.82(0.52,1.28)$ \\
\hline Black (race) & $407(17.9 \%)$ & $4858(11.3 \%)$ & $1.36(1.16,1.58)^{\mathrm{c}}$ \\
\hline Hispanic (race) & $100(4.4 \%)$ & $2224(5.2 \%)$ & $0.70(0.53,0.92)^{\mathrm{c}}$ \\
\hline Multiple races given (race) & $41(1.8 \%)$ & $363(0.8 \%)$ & $1.40(0.91,2.17)$ \\
\hline Race unknown (race) & $126(5.6 \%)$ & $830(1.9 \%)$ & $2.39(1.85,3.10)$ \\
\hline Weekday-8-5 (reg h) & $1010(44.5 \%)$ & $15,444(35.8 \%)$ & 1.00 (referent) \\
\hline Weekday-5-8 (non reg h) & $900(39.7 \%)$ & $15,115(35.0 \%)$ & $0.82(0.72,0.92)^{\mathrm{c}}$ \\
\hline Weekend & $359(15.8 \%)$ & $12,628(29.2 \%)$ & $0.39(0.33,0.46)^{\mathrm{c}}$ \\
\hline Median time until LWBS (min) & 112 (IQR: 58, 177) & - & - \\
\hline Median time until seen (min) & - & 9 (IQR: 0, 50) & - \\
\hline \multicolumn{4}{|l|}{ Chief reported complaint in ED } \\
\hline Abdominal/GI & $526(23.2 \%)$ & $6683(15.5 \%)$ & 1.00 (referent) \\
\hline Chest pain & $289(12.7 \%)$ & $7578(17.6 \%)$ & $0.66(0.54,0.80)^{\mathrm{c}}$ \\
\hline Gyn/Gu & $143(6.3 \%)$ & $1475(3.4 \%)$ & $1.10(0.85,1.43)$ \\
\hline HEENT & $76(3.4 \%)$ & $1329(3.1 \%)$ & $0.70(0.51,0.98)^{\mathrm{c}}$ \\
\hline Immune/infection & $145(6.4 \%)$ & $2744(6.4 \%)$ & $0.70(0.54,0.90)^{\mathrm{c}}$ \\
\hline Injury/pain & $562(24.8 \%)$ & $12,208(28.3 \%)$ & $0.66(0.56,0.78)^{\mathrm{c}}$ \\
\hline Neuro/headache & $321(14.2 \%)$ & $6306(14.6 \%)$ & $0.79(0.65,0.96)^{\mathrm{c}}$ \\
\hline Psych & $63(2.8 \%)$ & $1101(2.6 \%)$ & $0.67(0.47,0.97)^{\mathrm{c}}$ \\
\hline Other & $144(6.4 \%)$ & $3763(8.7 \%)$ & $0.58(0.45,0.75)^{\mathrm{c}}$ \\
\hline Mean heart rate (beats/min) $)^{a, b}$ & 86 (SD: 16) & 81 (SD: 16) & $1.20(1.15,1.26)^{\mathrm{c}}$ \\
\hline Mean respiratory rate (breaths/min) ${ }^{a}, \mathrm{~b}$ & 17 (SD: 4) & 17 (SD: 5) & $0.97(0.89,1.07)$ \\
\hline Mean blood $\mathrm{O}_{2}$ saturation $(\%)^{\mathrm{a}, \mathrm{b}}$ & 98 (SD: 3) & 97 (SD: 3) & $1.80(1.49,2.17)^{\mathrm{c}}$ \\
\hline Mean pain score $(0-10 \text { analog })^{\mathrm{a}}$ & $6.6(\mathrm{SD}: 3)$ & $5.6(\mathrm{SD}: 3)$ & $1.11(1.08,1.14)^{\mathrm{c}}$ \\
\hline Mean severity index ${ }^{a}$ & 3.2 (SD: 0.6$)$ & 3.0 (SD: 0.8$)$ & $1.59(1.40,1.81)^{\mathrm{c}}$ \\
\hline
\end{tabular}

$S D$ standard deviation, $I Q R$ interquartile range, $C C$ chief complaint

${ }^{a}$ Variables collected during nurse's triage were not available for the 301 patients who left prior to assessment. Presented adjusted odds ratios were based on fitting two models: one excluding variables with ' $a$ ' superscripts; and one including those variables. Estimates with 'a' superscript included those variables in the model

${ }^{\mathrm{b}}$ Adjusted odds ratio based on 10-unit increase in heart rate, 5-unit increase in respiratory rate and $\mathrm{O}_{2}$ saturation

${ }^{\mathrm{c}}$ Precision bounds excluded the null value
Table 2 Subsequent healthcare system utilizations for emergency department (ED) patients who left without being seen (LWBS) with an associated encounter within 3 weeks, $n=920$

\begin{tabular}{lc}
\hline Emergency department & $621(67.5 \%)$ \\
Clinic/urgent care & $253(27.5 \%)$ \\
$\begin{array}{l}\text { Other provider (specialty or other provider appoint- } \\
\text { ment) }\end{array}$ & $46(5.0 \%)$ \\
Associated ED bounce back within $72 \mathrm{~h}$ & $485(52.7 \%)$ \\
Associated hospital admission within 3 weeks & $128(13.9 \%)$ \\
\hline
\end{tabular}

Total study sample included 2269 LWBS during the reviewed year with 1349 not having a documented associated encounter within the local health system and excluded from this table admitted patients was $78.5 \mathrm{~h}$ (IQR: 41, 146), with additional descriptive data about these patients available in Supplemental Table 1. In addition, descriptive data about the 302 LWBS patients who left before nurse triage are available in Supplemental Table 2.

The exploratory examination on the relationship between the number of daily LWBS patients and ED daily census and/ or ED daily inpatient boarding revealed positive correlations. Overall, initial results suggest that every additional patient in census would increase the number of LWBS patients by 0.21 (95\% CI 0.18, 0.25); similarly, every additional patient in boarding would increase the number of LWBS patients by 0.27 (95\% CI $0.21,0.34)$. Estimates controlled for both 
Table 3 Characteristics of emergency department (ED) left without being seen patients stratified by documented associated visit within health system within 3 weeks following ED encounter status, $n=2269$

\begin{tabular}{|c|c|c|c|}
\hline Characteristics & $\begin{array}{l}\text { Associated visit in } \\
3 \text { weeks }(n=920)\end{array}$ & $\begin{array}{l}\text { No associated visit in } \\
3 \text { weeks }(n=1349)\end{array}$ & $\begin{array}{l}\text { Adjusted odds ratio } \\
(99 \% \mathrm{CI})\end{array}$ \\
\hline Mean age (years) & 42 (SD: 18) & 39 (SD: 17) & $1.08(1.01,1.15)^{\mathrm{c}}$ \\
\hline Male & $348(37.8 \%)$ & $484(35.9 \%)$ & $1.09(0.85,1.38)$ \\
\hline White (race) & $692(75.2 \%)$ & $868(64.3 \%)$ & 1.00 (referent) \\
\hline Asian (race) & $15(1.6 \%)$ & $20(1.5 \%)$ & $0.98(0.40,2.43)$ \\
\hline Black (race) & $140(15.2 \%)$ & $267(19.8 \%)$ & $0.68(0.50,0.93)^{\mathrm{c}}$ \\
\hline Hispanic (race) & $37(4.0 \%)$ & $63(4.7 \%)$ & $0.81(0.46,1.41)$ \\
\hline Multiple (race) & $15(1.6 \%)$ & $26(1.9 \%)$ & $0.75(0.32,1.78)$ \\
\hline Unknown (race) & $21(2.3 \%)$ & $105(7.8 \%)$ & $0.25(0.13,0.47)^{\mathrm{c}}$ \\
\hline Weekday (8 am-5 pm) & $448(48.7 \%)$ & $562(41.7 \%)$ & 1.00 (referent) \\
\hline Weekday (not 8 am-5 pm) & $353(38.4 \%)$ & $547(40.6 \%)$ & $0.87(0.67,1.11)$ \\
\hline Weekend & $119(12.9 \%)$ & $240(17.8 \%)$ & $0.63(0.45,0.89)^{\mathrm{c}}$ \\
\hline Median ED wait time (min) & 111 (IQR: 57, 177) & 114 (IQR: 59, 177) & $1.00(0.98,1.01)$ \\
\hline \multicolumn{4}{|l|}{ Chief complaint in ED } \\
\hline Abdominal/gastrointestinal & $214(23.3 \%)$ & $312(23.1 \%)$ & 1.00 (referent) \\
\hline Chest pain & $131(14.2 \%)$ & $158(11.7 \%)$ & $1.08(0.73,1.59)$ \\
\hline Gynecological/genitourinary & $71(7.7 \%)$ & $72(5.3 \%)$ & $1.51(0.92,2.48)$ \\
\hline HEENT & $17(1.9 \%)$ & $59(4.4 \%)$ & $0.41(0.19,0.87)^{\mathrm{c}}$ \\
\hline Immune/infection & $56(6.1 \%)$ & $89(6.6 \%)$ & $0.91(0.55,1.52)$ \\
\hline Injury/pain & $221(24.0 \%)$ & $341(25.3 \%)$ & $0.91(0.65,1.27)$ \\
\hline Neuro/headache & $125(13.6 \%)$ & $196(14.5 \%)$ & $0.91(0.62,1.33)$ \\
\hline Psychiatric & $19(2.1 \%)$ & $44(3.3 \%)$ & $0.65(0.30,1.38)$ \\
\hline Other & $63(6.9 \%)$ & $70(5.2 \%)$ & $1.15(0.70,1.90)$ \\
\hline Mean heart rate (beats/min) ${ }^{a}$ & 87 (SD: 16) & 86 (SD: 16) & $1.05(0.96,1.15)$ \\
\hline Mean respiratory rate (breaths $/ \mathrm{min})^{\mathrm{a}}$ & 17 (SD: 4) & 17 (SD: 4) & $1.03(0.88,1.21)$ \\
\hline Mean blood oxygen saturation $(\%)^{\mathrm{a}}$ & 97 (SD: 2) & 98 (SD: 3) & $0.96(0.64,1.42)$ \\
\hline Mean pain score ${ }^{\mathrm{a}}$ & 6.5 (SD: 3) & 6.6 (SD: 3) & $1.00(0.95,1.05)$ \\
\hline Mean severity index ${ }^{a}$ & 3.2 (SD: 0.5$)$ & 3.3 (SD: 0.6) & $0.99(0.73,1.34)$ \\
\hline
\end{tabular}

$S D$ standard deviation, IQR interquartile range, $C C$ chief complaint

${ }^{a}$ Variables collected during nurse's triage were not available for the 301 patients who left prior to assessment. Presented adjusted odds ratios were based on fitting two models: one excluding variables with ' $a$ ' superscripts; and one including those variables. Estimates with 'a' superscript included those variables in the model

${ }^{\mathrm{b}}$ Adjusted odds ratio based on 10-unit increase in heart rate, 5-unit increase in respiratory rate and $\mathrm{O}_{2}$ saturation, and 1-unit increase in age, pain and severity

${ }^{c}$ Precision bounds excluded the null value variables. These findings suggest that roughly five additional patients in census would lead to an additional patient leaving without being seen, as would roughly four additional boarding patients. These three variables are presented using heat maps in Supplemental Figures 1, 2, 3.

\section{Discussion}

This analysis by patients who LWBS from a single Midwestern ED over a year found a substantial number of patients continue to seek healthcare within the same organization, and specifically go on to have their LWBS concern addressed through other venues in the same organization.
While there remain concerns with LWBS effects on patients' care quality and outcomes, among other reasons to continue to reduce LWBS rates, these data can help inform administrative strategies to optimize health systems and improve efficiency. This finding builds on the limited data that exist from previous studies on subsequent healthcare utilization of LWBS patients [2]. The effect of specific efforts to mitigate LWBS rates as well as future studies comparing these retention rates to that of patients who did not LWBS are still necessary.

Patients who returned to the ED within $72 \mathrm{~h}$ were of particular interest in this study. These patients represent the subset of the LWBS population that should be especially concerning, as the multiple ED visits for the same unevaluated 
Table 4 Characteristics of emergency department left without being seen patients stratified return to the $\mathrm{ED}$ within $72 \mathrm{~h}$ for an associated concern, $n=2269$

\begin{tabular}{|c|c|c|c|}
\hline Characteristics & $\begin{array}{l}\text { Returned to the ED } \\
\text { w/in } 72 \mathrm{~h}(n=485)\end{array}$ & $\begin{array}{l}\text { Did not returned to the } \\
\text { ED w/in } 72 \mathrm{~h}(n=1784)\end{array}$ & $\begin{array}{l}\text { Adjusted odds ratio } \\
\text { (99\% CI) }\end{array}$ \\
\hline Mean age (years) & 43 (SD: 19) & 40 (SD: 17) & $1.09(1.01,1.18)^{\mathrm{c}}$ \\
\hline Male & $186(38.4 \%)$ & $646(36.2 \%)$ & $1.08(0.81,1.44)$ \\
\hline White (race) & $366(75.5 \%)$ & $1,194(66.9 \%)$ & 1.00 (referent) \\
\hline Asian (race) & $7(1.4 \%)$ & $28(1.6 \%)$ & $0.84(0.27,2.56)$ \\
\hline Black (race) & $79(16.3 \%)$ & $328(18.4 \%)$ & $0.83(0.57,1.20)$ \\
\hline Hispanic (race) & $17(3.5 \%)$ & $83(4.7 \%)$ & $0.73(0.36,1.48)$ \\
\hline Multiple (race) & $7(1.4 \%)$ & $34(1.9 \%)$ & $0.70(0.24,2.09)$ \\
\hline Unknown (race) & $9(1.9 \%)$ & $117(6.6 \%)$ & $0.26(0.10,0.64)^{\mathrm{c}}$ \\
\hline Weekday (8 am-5 pm) & $232(47.8 \%)$ & $778(43.6 \%)$ & 1.00 (referent) \\
\hline Weekday (not 8 am-5 pm) & $195(40.2 \%)$ & $705(39.5 \%)$ & $0.98(0.73,1.31)$ \\
\hline Weekend & $58(12.0 \%)$ & $301(16.9 \%)$ & $0.64(0.41,0.97)^{\mathrm{c}}$ \\
\hline Median ED wait time (mins) & 106 (IQR: 52, 168) & 113 (IQR: 60, 180) & $0.98(0.96,1.00)$ \\
\hline \multicolumn{4}{|l|}{ Chief complaint in ED } \\
\hline Abdominal/gastrointestinal & $125(25.8 \%)$ & $401(22.5 \%)$ & 1.00 (referent) \\
\hline Chest pain & $65(13.4 \%)$ & $224(12.6 \%)$ & $0.82(0.52,1.30)$ \\
\hline Gynecological/genitourinary & $34(7.0 \%)$ & $109(6.1 \%)$ & $1.04(0.58,1.85)$ \\
\hline HEENT & $7(1.4 \%)$ & $69(3.9 \%)$ & $0.30(0.10,0.86)^{\mathrm{c}}$ \\
\hline Immune/infection & $28(5.8 \%)$ & $117(6.6 \%)$ & $0.75(0.40,1.37)$ \\
\hline Injury/pain & $118(24.3 \%)$ & $444(24.9 \%)$ & $0.78(0.53,1.15)$ \\
\hline Neuro/headache & $59(12.2 \%)$ & $262(14.7 \%)$ & $0.71(0.44,1.12)$ \\
\hline Psychiatric & $12(2.5 \%)$ & $51(2.9 \%)$ & $0.77(0.32,1.87)$ \\
\hline Other & $37(7.6 \%)$ & $107(6.0 \%)$ & $0.99(0.56,1.75)$ \\
\hline Mean heart rate (beats/min) ${ }^{\mathrm{a}}$ & 88 (SD: 16$)$ & 86 (SD: 16$)$ & $1.07(0.96,1.19)$ \\
\hline Mean respiratory rate (breaths $/ \mathrm{min})^{\mathrm{a}}$ & 17 (SD: 4) & 17 (SD: 4) & $1.00(0.83,1.21)$ \\
\hline Mean blood oxygen saturation $(\%)^{\mathrm{a}}$ & 97 (SD: 2) & 98 (SD: 3) & $0.97(0.61,1.54)$ \\
\hline Mean pain score ${ }^{a}$ & 6.7 (SD: 3) & 6.5 (SD: 3) & $1.02(0.96,1.09)$ \\
\hline Mean severity index ${ }^{a}$ & $3.2(\mathrm{SD}: 0.6)$ & 3.3 (SD: 0.6) & $0.83(0.58,1.20)$ \\
\hline
\end{tabular}

$S D$ standard deviation, $I Q R$ interquartile range, $C C$ chief complaint

${ }^{a}$ Variables collected during nurse's triage were not available for the 301 patients who left prior to assessment. Presented adjusted odds ratios were based on fitting two models: one excluding variables with ' $a$ ' superscripts; and one including those variables. Estimates with 'a' superscript included those variables in the model

${ }^{\mathrm{b}}$ Adjusted odds ratio based on 10-unit increase in heart rate, 5-unit increase in respiratory rate and $\mathrm{O}_{2}$ saturation, and 1-unit increase in age, pain and severity

${ }^{\mathrm{c}}$ Precision bounds excluded the null value concern could result in preventable medical complications and high costs; however, they also represent a degree of confidence in the same institution by the patients to return and not seek emergency care elsewhere. The predictive factors of ED returns found in this study will be important considerations moving forward for EDs wishing to minimize the impact LWBS can have on their health system.

Patients who were admitted were much more likely to have their post-LWBS visit be to the ED rather than to a clinic, perhaps indicating the severity/progression of the index ED concern. Patients who LWBS prior to nursing triage subsequently had a lower rate of returning to the ED and being admitted to the hospital. While this may seem to suggest that these patients may be accurate in believing themselves to be well enough to leave prior to triage, we cannot conclude this given our inability to account for subsequent care received at other facilities within the area.

Given that a majority of patients who leave the ED are likely to seek subsequent care within the same system, this could impact providers in a variety of ways. Specifically, recent changes in health policies in the United States have increased support for Accountable Care Organizations (ACOs) and in turn incentivized healthcare systems to not only mitigate costly ED visits but also retain patients within their networks [9]. Patients leaving the ED without being seen may have historically represented a potential loss of revenue, but the opposite may be true in an ACO structure. Thus, substitution to non-ED care after LWBS may 
reduce total medical costs for a health system participating in an ACO. While this scenario is speculative, it becomes important to evaluate patient behavior after LWBS visits in order to understand the likelihood patients will still receive care and whether they will do so within the same healthcare system. Furthermore, this study may be a valuable baseline from which health care organizations' future ventures and responses to LWBS can be gauged in light of changing healthcare economics.

Further research is needed to compare the behaviors of the LWBS population to those who are fully evaluated in the ED. Differences in retention rates between these two populations could identify potential incremental effects of LWBS on a health system's market share, but the findings presented here remain valuable for identifying the post-LWBS course within a health system.

\section{Limitations}

This study focused on the health system's perspective on patient retention and subsequent provided healthcare after a patient left the ED without being seen, meaning that data could not be captured for patients who received care elsewhere. It did not intend to capture outcomes for all the LWBS patients, but rather focused on the characteristics and predictors surrounding those who continued to return to the same organization. Similarly, the presented study took place within a single region and therefore limits the generalizability of findings, as the demographics, infrastructure, and resources of other communities may impact a patient's postLWBS care utilization differently. For example, personnel at the study ED do not routinely attempt to contact LWBS patients for follow-up.

Patients who left prior to an initial nurse triage had a limited interaction with the study ED and often provided no data beyond a chief complaint. A nominal amount of patients contributed more than one LWBS encounter to the dataset. The potential dependencies between these encounters were not directly controlled for in models, but indirectly combatted using a smaller alpha value in calculated estimates.

This study looked at data pertaining to ED visits between February 1, 2019 and January 31, 2020, a period just months before the COVID-19 pandemic struck the United States. It is important to consider that EDs and health systems have gone through substantial changes during these unprecedented times and could potentially shift many dynamics such as LWBS rates and subsequent healthcare utilization.

Lastly, the impact of daily ED census and boarding on LWBS counts were examined as daily totals. This means the hour-to-hour variability throughout the course of the day was not examined and could contribute significantly to the relationship between ED inpatient boarding, which tends to peak in the afternoon-to-evening, and LWBS. Hourly data and possible correlations could be a valuable area of further study.

\section{Conclusions}

At least one-half of LWBS patients did not go untreated as they sought care related to the concerns by a provider within 3 weeks of the initial encounter. The high prevalence of ED returns within a narrow turnaround window highlights a missed opportunity to provide services to these patients during their initial encounter.

Supplementary Information The online version contains supplementary material available at https://doi.org/10.1007/s11739-021-02775-z.

Acknowledgements The study team would like to thank Vanessa Calderon for her assistance in acquiring components of the study data.

Authors' contribution NK and $\mathrm{CH}$ conceived the study, with HS helping in design of the study and obtain appropriate approval. NK and SC supervised the conduct of the study and data collection, primarily completed by NR as well as SD. HS and JH contributed data collection elements, provided statistical advice and analyzed data. NR drafted the original manuscript, with all others contributing significantly to its revision. NK takes responsibility for the paper as a whole.

Funding The authors received no external funding with regards to this study.

Data and material availability The datasets generated during and/or analyzed during the current study are available on reasonable request.

Code availability The SAS 9.4 custom codes used to generate the results of the current study are available on reasonable request.

\section{Declarations}

Conflict of interest The authors declare that they have no conflicts of interest.

Ethical approval Ethical approval was obtained from the local Institutional Review Board for this retrospective study.

Consent to participate Informed consent to participate was not obtained as both the authors and IRB agreed that it was not applicable given the retrospective nature of the current study.

Consent to publication Informed consent to publish was not obtained as both the authors and IRB agreed that it was not applicable given the retrospective nature of the current study.

\section{References}

1. Emergency Medical Treatment and Labor Act (EMTALA). Centers for medicare and medicaid services. https://www.cms.gov/ Regulations-and-Guidance/Legislation/EMTALA. Accessed 4 Aug 2020 
2. Kennedy M, Macbean CE, Brand C et al (2008) Review article: leaving the emergency department without being seen. Emerg Med Australas 20(4):306-313. https://doi.org/10.1111/j.17426723.2008.01103.x

3. Hsia RY, Asch SM, Weiss RE et al (2011) Hospital determinants of emergency department left without being seen rates. Ann Emerg Med. https://doi.org/10.1016/j.annemergmed.2011.01.009

4. Johnson M, Myers S, Wineholt J et al (2009) Patients who leave the emergency department without being seen. J Emerg Nurs 35(2):105-108. https://doi.org/10.1016/j.jen.2008.05.006

5. Tropea J, Sundararajan V, Gorelik A et al (2012) Patients who leave without being seen in emergency departments: an analysis of predictive factors and outcomes. Acad Emerg Med 19(4):439447. https://doi.org/10.1111/j.1553-2712.2012.01327.x

6. Sibbritt D, Isbister GK, Walker R (2006) Emergency department performance indicators that encompass the patient journey. Qual Manag Health Care 15(1):27-38. https://doi.org/10.1097/00019 514-200601000-00004

7. Rowe BH, Channan P, Bullard M et al (2006) Characteristics of patients who leave emergency departments without being seen. Acad Emerg Med 13(8):848-852. https://doi.org/10.1197/j.aem. 2006.01.028
8. Clarey AJ, Cooke MW (2011) Patients who leave emergency departments without being seen: literature review and English data analysis. Emerg Med J 29(8):617-621. https://doi.org/10. 1136/emermed-2011-200537

9. Shortell SM, Sehgal NJ, Bibi S et al (2015) An early assessment of accountable care organizations' efforts to engage patients and their families. Med Care Res Rev 72(5):580-604. https://doi.org/ $10.1177 / 1077558715588874$

10. Ding R, Jung JJ, Kirsch TD et al (2007) Uncompleted emergency department care: patients who leave against medical advice. Acad Emerg Med 14(10):870-876. https://doi.org/10.1197/j.aem.2007. 06.027

11. Wilson BJ, Zimmerman D, Applebaum KG et al (2013) Patients who leave before being seen in an urgent care setting. Eur J Emerg Med 20(6):420-424. https://doi.org/10.1097/MEJ.0b013e3283 $59167 \mathrm{e}$

Publisher's Note Springer Nature remains neutral with regard to jurisdictional claims in published maps and institutional affiliations. 